\title{
Positive culture rate and antimicrobial susceptibilities of Mycoplasma hominis and Ureaplasma urealyticum
}

\author{
Young-Soo Jang, Ji-Won Min, Yun-Sook Kim \\ Department of Obstetrics and Gynecology, Soonchunhyang University Cheonan Hospital, Soonchunhyang University College of Medicine, Cheonan, \\ Korea
}

\section{Objective}

To assess positive culture rate and antimicrobial susceptibilities of Mycoplasma hominis (MH) and Ureaplasma urealyticum (UU) in symptomatic general population and pregnant women admitted with preterm labor and premature rupture of membranes.

\section{Methods}

We retrospectively reviewed medical records of patients who have undergone culture test and antimicrobial susceptibilities at our center from January 2017 to April 2018. Patients with positive culture for $\mathrm{MH}$, UU, or both were included in this study.

\section{Results}

There were 200 patients who were eligible for enrollment. Of these patients, $34(17 \%)$ were pregnant women and $166(83 \%)$ were non-pregnant women. Of these 200 patients, positive culture results were as follows: $\mathrm{MH}$ only, $n=10(5 \%)$; UU only, $n=58(29 \%) ;$ and both $M H$ and UU, $n=36(18 \%)$. Susceptibilities of MH only to doxycycline, erythromycin, ciprofloxacin, and azithromycin were $100 \%, 10 \%, 40 \%$, and $0 \%$, respectively. Susceptibilities of UU only to doxycycline, erythromycin, ciprofloxacin, and azithromycin were $94.8 \%, 87.9 \%, 5.2 \%$, and $81 \%$, respectively. Susceptibilities of both MH and UU to doxycycline, erythromycin, ciprofloxacin, and azithromycin were $97.2 \%, 5.6 \%$, $11.1 \%$, and $11.1 \%$, respectively.

\section{Conclusion}

UU only was the leading causative pathogen for genitourinary infection in our study. MH only accounted for about one sixth of UU only infections. Doxycycline was still the best antibiotics as most patients with MH only, UU only, or both $\mathrm{MH}$ and UU positive culture showed susceptibility. For ciprofloxacin, less than $12 \%$ of those with UU only and both $\mathrm{MH}$ and UU culture positive results showed susceptibility.

Keywords: Mycoplasma hominis; Ureaplasma urealyticum; Culture rate; Antimicrobial susceptibility; Pregnancy

\section{Introduction}

Mycoplasma hominis (MH) and Ureaplasma urealyticum (UU) are facultative anaerobic pathogens that usually colonize the lower genitourinary tract $[1,2]$. Although most colonized women remain asymptomatic, vaginal colonization with $\mathrm{MH}, \mathrm{UU}$, or both are associated with an increased risk of developing certain pathogenic conditions and pregnancy complications, such as pelvic inflammatory disease, postpartum infection, infertility, premature rupture of membranes (PROM), preterm labor, preterm birth, and systemic neonatal infections [3-5]. Doxycycline and quinolones are drugs of
Received: 2018.06.16. Revised: 2018.11.25. Accepted: 2018.12.03. Corresponding author: Yun-Sook Kim

Department of Obstetrics and Gynecology, Soonchunhyang University Cheonan Hospital, Soonchunhyang University College of Medicine, 31 Soonchunhyang 6-gil, Dongnam-gu, Cheonan 31151, Korea

E-mail: drsook@schmc.ac.kr

https://orcid.org/0000-0001-8427-4006

Articles published in Obstet Gynecol Sci are open-access, distributed under the terms of the Creative Commons Attribution Non-Commercial License (http://creativecommons. org/licenses/by-nc/3.0/) which permits unrestricted non-commercial use, distribution, and reproduction in any medium, provided the original work is properly cited.

Copyright $\odot 2019$ Korean Society of Obstetrics and Gynecology 


\title{
Obstetrics \& Gynecology Science
}

\author{
Vol. 62, No. 2, 2019
}

choice [6-8]. However, obstetricians empirically use macrolides for treatment of pregnant women in many cases due to fear of fetal abnormalities $[9,10]$. Antimicrobial susceptibility of $\mathrm{MH}, \mathrm{UU}$, and both has changed with times. It varies with geographic region too [11-15]. Therefore, it is essential to know the latest antibiotic sensitivity in a particular region to ensure successful treatment. Such study regarding antimicrobial susceptibilities of $\mathrm{MH}, \mathrm{UU}$, and both in pregnant and non-pregnant women has not been reported in Korea yet. Therefore, the objective of this study was to investigate culture rates and the antimicrobial susceptibilities of $\mathrm{MH}, \mathrm{UU}$, and both $\mathrm{MH}$ and $\mathrm{UU}$ in pregnant and non-pregnant women residing in Cheonan city, a Western area in South Korea.

\section{Materials and methods}

Non-pregnant women had undergone $\mathrm{MH}$ and UU culture and antimicrobial susceptibility test for abnormal vaginal dis-

Table 1. Clinical profiles of non-pregnant women $(n=166)$

\begin{tabular}{lr}
\hline Characteristics & Values \\
\hline Age (yr) & $37.6 \pm 2.4$ \\
Parity & $3.3 \pm 0.2$ \\
Body mass index & $29.5 \pm 6.9$ \\
Symptom & \\
Foul odor (fish \& rotten) & $101(60.8)$ \\
Itching \& burning sensation & $45(27.1)$ \\
Abnormal color of discharge & $20(12.1)$ \\
Total & $166(100)$ \\
\hline
\end{tabular}

Values are presented as mean \pm standard deviation or number (\%).

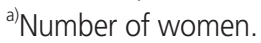

charge at our center from January 2017 to April 2018 were enrolled. In the non-pregnant group, tests were performed only for abnormal vaginal discharge, regardless of age, fertility, body mass index, underlying disease, or gynecologic disease. Abnormal vaginal discharge was considered present when the patient described having abnormal and bothersome discharge with respect to color, appearance, and odor as well as itching and burning sensations, necessitating examination and treatment (Table 1). In our study, we analyzed both UU and Ureaplasma parvum (UP) for ureaplasma infection. In the case of pregnant women, culture and antibiotic sensitivity tests were conducted only in the presence of a positive polymerase chain reaction test after admission for preterm labor or PROM. The patients-age ranged from 21 to 34 years, and culture was performed in women at more than 32 weeks of pregnancy. There were 30 cases of hospitalization because of preterm labor and 4 cases of hospitalization because of PROM. Women with multiple pregnancies were excluded from the study (Table 2).

Vaginal swabs were obtained from 200 women with a mean age of 37.7 years (range, 10-87 years) who were tested for the presence of $\mathrm{MH}$ and $\mathrm{UU}$ at Soonchunhyang University Cheonan Hospital between January 1, 2017, and April 30, 2018. Identification and antimicrobial susceptibilities of MU and UU were determined with a commercially available MYCOFAST Mycoplasma IST2 kit (bioMerieux, Macrcy-l'Etoile, France), as indicated by the manufacturer. Most hospitals in recent years use the same method despite using kits from different manufacturers. Briefly, the cotton swab included in the kit was inoculated in R1 transport medium, which could inhibit most gram-negative and grampositive bacteria. The inoculated R1 medium was vortexed rapidly and $3 \mathrm{~mL}$ was added to $\mathrm{R} 2$ growth medium contain-

Table 2. Clinical profiles of pregnant women $(n=34)$

\begin{tabular}{|c|c|c|c|}
\hline Characteristics & Total & Preterm birth & Premature rupture of membranes \\
\hline No. of women & 34 & 30 & 4 \\
\hline Age (yr) & $28.6 \pm 3.6$ & $28.7 \pm 4.3$ & $28.3 \pm 7.6$ \\
\hline Number with age $<25 \mathrm{yr}$ & 10 & 10 & 0 \\
\hline Gestational weeks at entry (wk) & $32.4 \pm 3.5$ & $32.5 \pm 4.1$ & $32.3 \pm 8.4$ \\
\hline Nulliparous & 28 & 25 & 3 \\
\hline Body mass index at entry of $<18.5 \mathrm{~kg} / \mathrm{m}^{2}$ & 6 & 5 & 1 \\
\hline History of preterm birth & 9 & 8 & 1 \\
\hline Cervical cerclage & 4 & 3 & 1 \\
\hline
\end{tabular}




\section{Obstetrics \& Gynecology Science}

Young-Soo Jang, et al. Culture and antimicrobial susceptibilities of Mycoplasma and Ureaplasma

ing $1 \mathrm{~mL}$ lyophilized urea/arginine broth. After reconstitution and shaking, $55 \mu \mathrm{L}$ was dispensed into each of 22 test wells on the strip. Two drops of mineral oil were added to each well. The remainder of the R2 medium and the inoculated strip were then incubated at $37^{\circ} \mathrm{C}$ and observed for color changes at 24 and 48 hours. Antimicrobial susceptibility testing included doxycycline, josamycin, ofloxacin, erythromycin, tetracycline, ciprofloxacin, azithromycin, clarithromycin, and pristinamycin.

The development or absence of red color at relevant part of the strip provided an index of resistance or susceptibility to each antimicrobial agent according to guidelines of the Clinical \& Laboratory Standards Institute. The breakpoints for the antimicrobials tested are as follows: tetracycline susceptible $S \leq 4$, resistant $R \geq 8$; doxycycline $S \leq 4, R \geq 8$; azithromycin $S \leq 0.12, R \geq 4$; clarithromycin $S \leq 1, R \geq 4$; erythromycin $S \leq 1$, $R \geq 4$; josamycin $S \leq 2, R \geq 8$; ciprofloxacin $S \leq 1, R \geq 2$; ofloxacin $S \leq 1, R \geq 4$; pristinamycin $S \leq 1, R \geq 2$. Per study protocol, these patients had not received any previous treatment with antibiotics for at least 6 weeks. Statistical analyses were performed using SPSS Statistics ver. 21 (SPSS, Chicago, IL, USA). Correlation was analyzed using $\chi^{2}$ test and Fisher's exact test. $P$ values $<0.05$ were considered statistically significant.

\section{Results}

The condition was assessed in 200 patients, including 34 (17\%) pregnant women and 166 (83\%) non-pregnant women. Of a total of 200 patients, positive culture results are as follows: $\mathrm{MH}$ only, $\mathrm{n=10}(5 \%)$; UU only, $\mathrm{n}=58$ (29\%); and both $\mathrm{MH}$ and $\mathrm{UU}, \mathrm{n}=36$ (18\%). The susceptibilities of $\mathrm{MH}$ only to doxycycline, erythromycin, ciprofloxacin, and azithromycin were $100 \%, 10 \%, 40 \%$, and $0 \%$, respectively. The susceptibilities of UU only to doxycycline, erythromycin, ciprofloxacin, and azithromycin were 94.8\%, 87.9\%, 5.2\%, and $81 \%$, respectively. The susceptibilities of both $\mathrm{MH}$ and UU to doxycycline, erythromycin, ciprofloxacin, and azithromycin were $97.2 \%, 5.6 \%, 11.1 \%$, and $11.1 \%$, respectively (Table 3).

In 166 non-pregnant women, the positive culture results were as follows: $\mathrm{MH}$ only, $\mathrm{n}=10$ (6\%); UU only, $\mathrm{n}=49$ (29.5\%); and both $\mathrm{MH}$ and $\mathrm{UU}, \mathrm{n}=32$ (19.3\%). The susceptibilities of $\mathrm{MH}$ only to doxycycline, erythromycin, ciprofloxacin, and azithromycin were $100 \%, 10 \%, 40 \%$, and $0 \%$, respectively. The susceptibilities of UU only to doxycycline, erythromycin, ciprofloxacin, and azithromycin were 93.9\%, 89.8\%, 6.1\%, and $79.6 \%$, respectively. The susceptibilities of both $\mathrm{MH}$ and UU to doxycycline, erythromycin, ciprofloxacin, and azithromycin were $96.9 \%, 6.3 \%, 12.5 \%$, and $12.5 \%$, respectively (Table 4).

In 34 pregnant women, positive culture results were as follows: $\mathrm{MH}$ only, $\mathrm{n}=0(0 \%)$; UU only, $\mathrm{n}=9(26.5 \%)$; and both $\mathrm{MH}$ and $\mathrm{UU}, \mathrm{n}=4$ (11.8\%). The susceptibilities of $\mathrm{MH}$ only to doxycycline, erythromycin, ciprofloxacin, and azithromycin were $0 \%, 0 \%, 0 \%$, and $0 \%$, respectively. The susceptibilities of UU only to doxycycline, erythromycin, ciprofloxacin, and azithromycin were $100 \%, 77.8 \%, 0 \%$, and $88.9 \%$, respectively. The susceptibilities of both $\mathrm{MH}$ and $\mathrm{UU}$ to doxycycline, erythromycin, ciprofloxacin, and azithromycin were 100\%, $0 \%, 0 \%$, and $0 \%$, respectively (Table 5 ).

\section{Discussion}

MH only infection accounted for about one-sixth of UU in-

Table 3. Antimicrobial susceptibilities (\%) of Mycoplasma hominis and Ureaplasma urealyticum for all patients ( $\mathrm{n}=200)$

\begin{tabular}{|c|c|c|c|c|c|c|}
\hline \multirow{2}{*}{ Antimicrobials } & \multicolumn{2}{|c|}{ MH only $(n=10,5 \%)$} & \multicolumn{2}{|c|}{ UU only $(n=58,29 \%)$} & \multicolumn{2}{|c|}{$M H+U U(n=36,18 \%)$} \\
\hline & $\mathrm{S}$ & $\mathbf{R}$ & $\mathbf{S}$ & $\mathbf{R}$ & $\mathbf{S}$ & $\mathbf{R}$ \\
\hline Doxycycline & $10(100)$ & $0(2.2)$ & $55(94.8)$ & $3(5.2)$ & $35(97.2)$ & $1(2.8)$ \\
\hline Erythromycin & $1(10)$ & $9(90)$ & $51(87.9)$ & $7(12.1)$ & $2(5.6)$ & $34(94.4)$ \\
\hline Ciprofloxacin & $4(40)$ & $6(60)$ & $3(5.2)$ & $55(94.8)$ & $4(11.1)$ & $32(88.9)$ \\
\hline Azithromycin & $0(0)$ & $10(100)$ & $47(81)$ & $41(19)$ & $4(11.1)$ & 32 (88.9) \\
\hline
\end{tabular}

Values are presented as number (\%). The breakpoints (mg/L) according to Clinical \& Laboratory Standards Institute were as follows: doxycycline $S \leq 4, R \geq 8$; erythromycin $S \leq 1, R \geq 4$; ciprofloxacin $S \leq 1, R \geq 2$.

S, susceptible; R, resistant; MH, Mycoplasma hominis; UU, Ureaplasma urealyticum. 


\title{
Obstetrics \& Gynecology Science
}

\author{
Vol. 62, No. 2, 2019
}

Table 4. Antimicrobial susceptibilities (\%) of Mycoplasma hominis and Ureaplasma urealyticum for non-pregnant women ( $\mathrm{n=166)}$

\begin{tabular}{|c|c|c|c|c|c|c|}
\hline \multirow{2}{*}{ Antimicrobials } & \multicolumn{2}{|c|}{ MH only $(n=10,6.0 \%)$} & \multicolumn{2}{|c|}{ UU only $(n=49,29.5 \%)$} & \multicolumn{2}{|c|}{$M H+U U(n=32,19.3 \%)$} \\
\hline & $S$ & $\mathbf{R}$ & $\mathrm{S}$ & $\mathbf{R}$ & $\mathrm{S}$ & $\mathbf{R}$ \\
\hline Doxycycline & $10(100)$ & $0(0)$ & $46(93.9)$ & $3(6.1)$ & $31(96.9)$ & $1(3.1)$ \\
\hline Erythromycin & $1(10)$ & $9(90)$ & $44(89.8)$ & $5(10.2)$ & $2(6.3)$ & $30(93.8)$ \\
\hline Ciprofloxacin & $4(40)$ & $6(60)$ & $3(6.1)$ & $46(93.9)$ & $4(12.5)$ & $28(87.5)$ \\
\hline Azithromycin & $0(0)$ & $10(100)$ & $39(79.6)$ & $10(20.4)$ & $4(12.5)$ & $28(87.5)$ \\
\hline
\end{tabular}

Values are presented as number (\%). The breakpoints ( $\mathrm{mg} / \mathrm{L}$ ) according to Clinical \& Laboratory Standards Institute were as follows: doxycycline $S \leq 4, R \geq 8$; erythromycin $S \leq 1, R \geq 4$; ciprofloxacin $S \leq 1, R \geq 2$.

S, susceptible; R, resistant; $\mathrm{MH}$, Mycoplasma hominis; UU, Ureaplasma urealyticum.

Table 5. Antimicrobial susceptibilities (\%) of Mycoplasma hominis and Ureaplasma urealyticum for pregnant women ( $\mathrm{n}=34)$

\begin{tabular}{|c|c|c|c|c|c|c|}
\hline \multirow{2}{*}{ Antimicrobials } & \multicolumn{2}{|c|}{ MH only $(n=0,0 \%)$} & \multicolumn{2}{|c|}{ UU only $(n=9,26.5 \%)$} & \multicolumn{2}{|c|}{$M H+U U(n=4,11.8 \%)$} \\
\hline & $\mathbf{S}$ & $\mathbf{R}$ & $\mathbf{S}$ & $\mathbf{R}$ & $\mathbf{S}$ & $\mathbf{R}$ \\
\hline Doxycycline & $0(0)$ & $0(0)$ & $9(100)$ & $0(0)$ & $4(100)$ & $0(0)$ \\
\hline Erythromycin & $0(0)$ & $0(0)$ & $7(77.8)$ & $2(22.2)$ & $0(0)$ & $4(100)$ \\
\hline Ciprofloxacin & $0(0)$ & $0(0)$ & $0(0)$ & $9(100)$ & $0(0)$ & $4(100)$ \\
\hline Azithromycin & $0(0)$ & $0(0)$ & $8(88.9)$ & $1(11.1)$ & $0(0)$ & $4(100)$ \\
\hline
\end{tabular}

Values are presented as number (\%). The breakpoints (mg/L) according to Clinical \& Laboratory Standards Institute were as follows: doxycycline $S \leq 4, R \geq 8$; erythromycin $S \leq 1, R \geq 4$; ciprofloxacin $S \leq 1, R \geq 2$.

S, susceptible; R, resistant; MH, Mycoplasma hominis; UU, Ureaplasma urealyticum.

fections in this study. The study population seemed to be mainly infected with UU. Doxycycline was still the best antibiotic because those with positive culture results for UU only, $\mathrm{MH}$ only, and both $\mathrm{MH}$ and UU showed susceptibilities. More than $80 \%$ of UU infections alone were sensitive to erythromycin and azithromycin, whereas less than $12 \%$ of $\mathrm{MH}$ and both $\mathrm{MH}$ and $\mathrm{UU}$ infections were sensitive to erythromycin and azithromycin. The susceptibility of $\mathrm{UU}$ only and both $\mathrm{MH}$ and UU to ciprofloxacin was only less than $12 \%$.

In this study, the incidence of $\mathrm{MH}, \mathrm{UU}$, both $\mathrm{MH}$ and $\mathrm{UU}$ infection and antimicrobial susceptibilities were similar between pregnant and non-pregnant women.

Organisms other than Chlamydia trachomatis and Neisseria gonorrhea, particularly UU, may be detected more frequently in patients with pelvic inflammatory disease in a Korean study. In addition, identification of MH may be important in female health problems such as Fitz-Hugh-Curtis syndrome [16]. MH and UU infection can cause urethritis and increase the risk of vaginitis, abnormal vaginal bleeding, and foul vaginal odor. $\mathrm{MH}$, like $\mathrm{UU}$, is commonly found in the urinary and genital tracts. In most cases, people with $\mathrm{MH}$ or UU infection do not have any symptoms. If there are any symptoms, they include difficulty and pain during urination, and discharge of fluid from the urethra. In women, the symptoms are vaginal discharge and dysuria. In men, the symptoms are urethritis, dysuria, and discharge. Besides symptoms, an indication for a laboratory test is a high-risk sexual behavior. All sexual contacts within the preceding 6 months of the onset of symptoms or diagnosis should ideally be evaluated, tested, and treated. Test-of-cure samples should be collected no earlier than 3 weeks after the start of treatment [17].

The major problem is that pregnant women infected with both $\mathrm{MH}$ and $\mathrm{UU}$ are not sensitive to erythromycin or azithromycin at all. The role of $\mathrm{MH}$ and $\mathrm{UU}$ in premature births may be related to their ability to induce inflammatory cytokines, thereby triggering pathways leading to preterm labor. The role of $\mathrm{MH}$ and $\mathrm{UU}$ in adverse pregnancy outcomes is increasingly accepted. However, the sole presence of these microorganisms in the vaginal flora might be insufficient to cause pathological issues. Other factors such as bacterial vaginosis or cervical incompetence may be additionally needed to induce preterm birth [18]. According to a Russian study, josamycin was inferior to azithromycin in terms of a number of pharmacologic parameters, compliance, and safety. Therefore, josamycin should not be used in pregnant women [19]. Doxycycline became immediately popular after 


\section{Obstetrics \& Gynecology Science}

Young-Soo Jang, et al. Culture and antimicrobial susceptibilities of Mycoplasma and Ureaplasma

its Food and Drug Administration approval in 1967 because of its simplified once (or twice)-a-day dosage regimen rather than the 4 times daily dosing scheme for tetracycline. Doxycycline was developed after tetracyclines had been labeled as potentially harmful because of severe adverse effects, including teratogenicity, permanent yellowish-brown teeth discoloration after in utero exposure and in children under 8 years of age, and, very rarely, fatal hepatotoxicity in pregnant women [20]. There is no evidence in the current literature of any human teratogenicity following the use of doxycycline during pregnancy [21]. Cumulative evidence suggests that the use of doxycycline during the first trimester is not associated with increased risk in the growing fetus [22]. Although many studies have reported that the use of doxycycline in early pregnancy has a low risk of causing malformations, there is insufficient data to conclude that there is no danger; thus, more research is needed.

Among pregnant women with preterm labor, the duration of pregnancy was prolonged in $\mathrm{MH}$ - and $\mathrm{UU}$-negative group than in positive groups [23]. Prepregnancy and early pregnancy screening for genital mycoplasmas and subsequent treatment may reduce preterm deliveries [24]. In our study, erythromycin and azithromycin were still active against UU. However, quinolone derivatives such as ciprofloxacin were inactive against UU. Only doxycycline was active against both $\mathrm{MH}$ and UU.

Compared to that in previous studies, the susceptibility to ciprofloxacin and azithromycin was significantly reduced in UU only and both $\mathrm{MH}$ and UU infections in this study. However, erythromycin and azithromycin were inactive against $\mathrm{MH}$. Although small in number, the positive culture rates of $\mathrm{MH}, \mathrm{UU}$, and both $\mathrm{MH}$ and $\mathrm{UU}$ in pregnant women in Cheonan with preterm labor or PROM were $0 \%, 26.5 \%$ and $11.8 \%$, respectively. In 2009, Koh et al. [25] reported that $44.2 \%$ of pregnant women in Jinju had genital mycoplasma infection. In 2004, Kim et al. [26] reported that infection with $\mathrm{MH}$ was a significant factor in preterm labor and PROM. Further, in 2014, Kwak et al. [27] reported that the prevalence of positive vaginal fluid cultures for genital mycoplasma was 62.5\% (112/179). This group included 99 patients infected with only UU and 13 patients infected with both $\mathrm{MH}$ and UU. No patients were found to be infected with only $\mathrm{MH}$. Compared with patients only positive for UU, patients with positive for both organisms showed significantly decreased gestational age at birth and neonatal birth weight, and significant increases in the incidences of preterm birth, neonatal intensive care unit admissions and histologic chorioamnionitis [27]. In pregnant women, $\mathrm{UU}$ and both $\mathrm{MH}$ and UU infections were resistant to ciprofloxacin but susceptible to doxycyline. Characteristically, the susceptibility of UU to erythromycin and azithromycin was $77.8 \%$ and $88.9 \%$, respectively. Azithromycin is the empirical treatment regimen for pregnant women in our geographic region.

In our symptomatic non-pregnant women $(n=166)$, the positive culture rate was $6 \%$ for $\mathrm{MH}, 29.5 \%$ for $\mathrm{UU}$, and $19.3 \%$ for both $\mathrm{MH}$ and UU. In 2016, Kwon et al. [28] reported that the positive culture rate in symptomatic nonpregnant women $(n=186)$ from vaginal swab sample was $11.8 \%$ for $\mathrm{MH}$ and $65.6 \%$ for $\mathrm{UU}$. These infection rates are much higher than those in our study. However, the $\mathrm{MH}$ infection rate was one-sixth of the UU infection rate, which is similar to our study. UU was still the leading causative pathogen for genitourinary infection in our study, as in other studies [29]. The use of empirical antibiotics can be ineffective owing to resistance to antibiotics. It is difficult to establish common guidelines for empirical treatment of genital $\mathrm{MH}$ and $\mathrm{UU}$ infections. With respect to the value of this research, we examined symptomatic patients and determined the antimicrobial susceptibilities for genital MH only, UU only, and both $\mathrm{MH}$ and $\mathrm{UU}$ in Cheonan, which can represent a specific geographic region, rather than the whole country. Nevertheless, we have achieved the following 2 important findings. First, compared with previous studies, the susceptibility to ciprofloxacin was significantly reduced when UU only and both $\mathrm{MH}$ and $\mathrm{UU}$ infections were present. Moreover, the susceptibility to azithromycin was significantly reduced when $\mathrm{MH}$ only and both $\mathrm{MH}$ and $\mathrm{UU}$ infections were present. Therefore, the empirical use of ciprofloxacin and azithromycin should be avoided in patients infected with $\mathrm{MH}, \mathrm{UU}$, and both $\mathrm{MH}$ and UU. Second, the MH infection rate was about one-sixth of the UU infection rate, and antibiotics other than doxycycline and ciprofloxacin have less than 10\% susceptibility. Efforts should be made to reduce the rate of $\mathrm{MH}$ and $\mathrm{UU}$ infection by conducting epidemiological studies. Planning pregnancy is the best way to treat infection before pregnancy. However, if only doxycycline is effective during pregnancy, much attention should be paid to which antibiotics should be used.

A nationwide survey may enable us to establish new guidelines for treating genital mycoplasma infections in Korea. 


\section{Obstetrics \& Gynecology Science}

Vol. 62, No. 2, 2019

This study collected many patients over the past 16 months between January 1, 2017, and April 30, 2018, with age ranging from 10 to 87 years. Empirical treatment without isolation or identification of genital $\mathrm{MH}$ and $\mathrm{UU}$ would fail in many cases. In vitro determination of antimicrobial susceptibility of genital $\mathrm{MH}$ and $\mathrm{UU}$ in each clinical case is required to avoid therapeutic failures.

\section{Acknowledgements}

The authors are grateful to Soonchunhyang University Cheonan Hospital for its assistance and encouragement.

\section{Conflict of interest}

No potential conflict of interest relevant to this article was reported.

\section{Ethical approval}

The study was approved by the Soonchunhyang University Cheonan Hospital Ethics Committee of the institution (elRB) (IRB No. 2018-05-049) and performed in accordance with principles of the Declaration of Helsinki. Written informed consents were obtained.

\section{Patient consent}

The patients provided written informed consent for publication and the use of their images.

\section{References}

1. Kilic D, Basar MM, Kaygusuz S, Yilmaz E, Basar H, Batislam E. Prevalence and treatment of Chlamydia trachomatis, Ureaplasma urealyticum, and Mycoplasma hominis in patients with non-gonococcal urethritis. Jpn J Infect Dis 2004;57:17-20.

2. Krohn MA, Hillier SL, Nugent RP, Cotch MF, Carey JC, Gibbs RS, et al. The genital flora of women with in- traamniotic infection. J Infect Dis 1995;171:1475-80.

3. Koch A, Bilina A, Teodorowicz L, Stary A. Mycoplasma hominis and Ureaplasma urealyticum in patients with sexually transmitted diseases. Wien Klin Wochenschr 1997;109:584-9.

4. McDonald HM, O'Loughlin JA, Jolley PT, Vigneswaran $R$, McDonald PJ. Changes in vaginal flora during pregnancy and association with preterm birth. J Infect Dis 1994;170:724-8.

5. Waites KB, Rudd PT, Crouse DT, Canupp KC, Nelson KG, Ramsey $C$, et al. Chronic Ureaplasma urealyticum and Mycoplasma hominis infections of central nervous system in preterm infants. Lancet 1988;1:17-21.

6. Arai S, Gohara Y, Kuwano K, Kawashima T. Antimycoplasmal activities of new quinolones, tetracyclines, and macrolides against Mycoplasma pneumoniae. Antimicrob Agents Chemother 1992;36:1322-4.

7. Hannan PC. Comparative susceptibilities of various AIDS-associated and human urogenital tract mycoplasmas and strains of Mycoplasma pneumoniae to 10 classes of antimicrobial agent in vitro. J Med Microbiol 1998;47:1115-22.

8. Kenny GE, Cartwright FD. Susceptibilities of Mycoplasma hominis and Ureaplasma urealyticum to two new quinolones, sparfloxacin and WIN 57273. Antimicrob Agents Chemother 1991;35:1515-6.

9. Ye Y, Tu S, Li H. Clinic intervention study on urogenital mycoplasma infection of pregnant women. Zhonghua Liu Xing Bing Xue Za Zhi 2001;22:293-5.

10. Ullmann U, Schubert $S$, Krausse R. Comparative in-vitro activity of levofloxacin, other fluoroquinolones, doxycycline and erythromycin against Ureaplasma urealyticum and Mycoplasma hominis. J Antimicrob Chemother 1999;43 Suppl C:33-6.

11. Lister PJ, Balechandran T, Ridgway GL, Robinson AJ. Comparison of azithromycin and doxycycline in the treatment of non-gonococcal urethritis in men. J Antimicrob Chemother 1993;31 Suppl E:185-92.

12. Romanowski B, Talbot H, Stadnyk M, Kowalchuk P, Bowie WR. Minocycline compared with doxycycline in the treatment of nongonococcal urethritis and mucopurulent cervicitis. Ann Intern Med 1993;119:16-22.

13. Stamm WE, Hicks CB, Martin DH, Leone P, Hook EW 3rd, Cooper RH, et al. Azithromycin for empirical treatment of the nongonococcal urethritis syndrome in men. 


\section{Obstetrics \& Gynecology Science}

Young-Soo Jang, et al. Culture and antimicrobial susceptibilities of Mycoplasma and Ureaplasma

A randomized double-blind study. JAMA 1995;274:5459.

14. Roberts MC, Kenny GE. Dissemination of the tetM tetracycline resistance determinant to Ureaplasma urealyticum. Antimicrob Agents Chemother 1986;29:350-2.

15. Leng Z, Riley DE, Berger RE, Krieger JN, Roberts MC. Distribution and mobility of the tetracycline resistance determinant tetQ. J Antimicrob Chemother 1997;40:5519.

16. Lee GH, Kim HJ, Park CH, Jeon YJ, Choi HJ, Lee HN, et al. Frequency of the causative organisms in PID and PHCS. Infect Chemother 2012;44:362-3.

17. Combaz-Söhnchen N, Kuhn A. A systematic review of Mycoplasma and Ureaplasma in urogynaecology. Geburtshilfe Frauenheilkd 2017;77:1299-303.

18. Capoccia R, Greub G, Baud D. Ureaplasma urealyticum, Mycoplasma hominis and adverse pregnancy outcomes. Curr Opin Infect Dis 2013;26:231-40.

19. Khrianin AA, Reshetnikov OV. Is it safe to use josamycin in the obstetrics practice in Russia? Antibiot Khimioter 2007;52:32-6.

20. Whalley PJ, Adams RH, Combes B. Tetracycline toxicity in pregnancy. Liver and pancreatic dysfunction. JAMA 1964; 189:357-62.

21. Cooper WO, Hernandez-Diaz S, Arbogast PG, Dudley JA, Dyer SM, Gideon PS, et al. Antibiotics potentially used in response to bioterrorism and the risk of major congenital malformations. Paediatr Perinat Epidemiol 2009;23:18-28.

22. Horne HW Jr, Kundsin RB. The role of mycoplasma among 81 consecutive pregnancies: a prospective study.
Int J Fertil 1980;25:315-7.

23. Wasiela M, Krzemiński Z, Hanke W, Kalinka J. Association between genital mycoplasmas and risk of preterm delivery. Med Wieku Rozwoj 2003;7 Suppl 1:211-6.

24. Park JK, Shin JK, Choi W, Lee SA, Lee JH, Paik WY. Mycoplasma infection of cervicovaginal fluid in women with preterm labor. Korean J Perinatol 2005;16:128-36.

25. Koh E, Kim S, Kim IS, Maeng KY, Lee SA. Antimicrobial Susceptibilities of Ureaplasma urealyticum and Mycoplasma hominis in Pregnant Women. Korean J Clin Microbiol 2009;12:159-62.

26. Kim SY, Lee YJ, Huh M, Lee SH, Park IY, Ahn HY, et al. Analysis of Mycoplasma hominis and Ureaplasma urealyticum infection in preterm labor and PROM patients. Korean J Obstet Gynecol 2004;47:1469-73.

27. Kwak DW, Hwang HS, Kwon JY, Park YW, Kim YH. Coinfection with vaginal Ureaplasma urealyticum and Mycoplasma hominis increases adverse pregnancy outcomes in patients with preterm labor or preterm premature rupture of membranes. J Matern Fetal Neonatal Med 2014;27:333-7.

28. Kwon OJ, Lim YK, Oh SM, Kim TH, Choi HS, Lee SJ. Prevalence and antimicrobial susceptibility of Mycoplasma hominis, Ureaplasma urealyticum and Ureaplasma parvum in individuals with or without symptoms of genitourinary infections. Lab Med Online 2016;6:79-87.

29. Lee HH, Ju KS, Lee KH, Won NH. Detection of Chlamydia trachomatis, Mycoplasma hominis and Ureaplasam urealyticum in the cervical swab and paraffin tissue with female genital tract infection. Korean J Obstet Gynecol 1999;42:549-55. 